\title{
Relatório de pareceristas da Revista de Sociologia e Política em 2011
}

Board of Reviewers of Revista de Sociologia e Política in 2011

Relación de evaluadores de la Revista de Sociologia e Política en 2011

Adriano Oliveira (UFPE) ad hoc

Alessandro André Leme (UFU) ad hoc

Alexandre K. Veronese Aguiar (UFF) ad hoc

Alexsandro Eugênio Pereira (UFPR)

Álvaro Augusto de Borba Barreto (Ufpel) ad hoc

Ana Rodrigues Cavalcanti Alves (UFPE) ad hoc

André Macedo Duarte (UFPR) ad hoc

André Vitor Singer (USP) ad hoc

Andréa Benetti Carvalho de Oliveira (UFPR) ad hoc

Antonio Sérgio Carvalho Rocha (Unifesp) ad hoc

Arnaldo José da Luz (UFPR) ad hoc

Bruno Bolognesi (Ufscar) ad hoc

Carla Almeida (UEM) ad hoc

Carla Villalta (Universidad de Buenos Aires, Argentina) ad hoc

Carlos Augusto Mello Machado (UnB) ad hoc

Carlos Eduardo Sell (UFSC) ad hoc

Carlos Schmidt Arturi (UFRGS)

Carlos Zacarias Sena Jr. (UFBA) ad hoc

Carolina Moulin Aguiar (PUC-RJ) ad hoc

Celene Tonela (UEM) ad hoc

César Barreira (UFC) ad hoc

Christian Edward Cyril Lynch (UFF) ad hoc

Christiane Jalles de Paula (FGV) ad hoc

Claudia Anzorena (Instituto de Ciencias Humanas, Sociales y Ambientales - Argentina) ad hoc

Clóvis Roberto Zimmermann (UFRB) ad hoc

Cristiano Fonseca Monteiro (UFF) ad hoc

Daniel Arias Vazquez (Unifesp) ad hoc

Daniel de Mendonça (Ufpel) ad hoc

Daniel Gustavo Mocelin (UFRGS) ad hoc

Daniela Gabbay (FGV-SP) ad hoc

Daniela Resende Archanjo (UFPR) ad hoc

Danielly Silva Ramos Becard (UnB)

Danilo Enrico Martuscelli (Unicamp) ad hoc

Débora Alves Maciel (Unifesp) ad hoc

Denise Paiva Ferreira (UFG) ad hoc

Doacir Gonçalves de Quadros (UFPR) ad hoc

Edemar Rotta (UFFS) ad hoc

Ednaldo Aparecido Ribeiro (UEM) ad hoc

Eduardo Cerqueira Battitucci (Fundação João Pinheiro) ad hoc

Eli Diniz (UFRJ) ad hoc

Emerson Maione de Souza (UFF) ad hoc

Emerson Urizzi Cervi (UFPR)

Érica Simone Resende (UFRJ) ad hoc

Érico Esteves Duarte (UFRGS) ad hoc

Ernesto Seidl (UFS) ad hoc

Erni José Seibel (UFSC) ad hoc

Eugênio Diniz (PUC-MG) ad hoc 
Fabiano Engelmann (UFRGS) ad hoc

Fabrício Ricardo de Limas Tomio (UFPR)

Feliciano de Sá Guimarães (FGV) ad hoc

Felipe Asensi (FGV) ad hoc

Fernando Baptista Leite (UFPR) ad hoc

Fernando Lattman Weltman (FGV) ad hoc

Fernando Salla (USP) ad hoc

Flávia Biroli (UnB) ad hoc

Flávia Braga Vieira (UFRRJ) ad hoc

Flávio Ramos (Univali) ad hoc

Francisco Carlos Palomanes Martinho (USP) ad hoc

Francisco de Assis Brandão dos Reis (UFPE) ad hoc

Frederico Normanha Ribeiro de Almeida (Univers. São Judas Tadeu) ad hoc

Gabriel Eduardo Vitullo (UFRN) ad hoc

Gabriel Kessler (Universidad Nacional de La Plata, Argentina) ad hoc

Gabriela da Silva Tarouco (UFPE) ad hoc

George Avelino (FGV) ad hoc

Geralda Luiza de Miranda (IPEA) ad hoc

Glauco Avelino Sampaio Oliveira (Ministério da Fazenda) ad hoc

Gustavo André Aveline Müller (UFSM) ad hoc

Gustavo Biscaia de Lacerda (UFPR)

Hamilton Garcia (UENF) ad hoc

Helcimara de Souza Telles (UFMG) ad hoc

Helena da Motta Salles (UFJF) ad hoc

Henri Acselrad (UFRJ) ad hoc

Igor Gastal Grill (UFMA) ad hoc

Inês Patrício (UFF) ad hoc

Ironildes Bueno da Silva (UnB) ad hoc

Ivann Carlos Lago (UFFS) ad hoc

Jacqueline Sinhoretto (Ufscar) ad hoc

Jaqueline Porto Zulini (USP) ad hoc

Javier Alberto Vadell (PUC-MG) ad hoc

Jevuks Matheus de Araújo (UFPE) ad hoc

Joana Vargas (UFRJ) ad hoc

João Carlos Amoroso Botelho (UFG) ad hoc

João Roberto Martins Filho (Ufscar)

Jorge Adriano Lubenow (UFPI) ad hoc

Jorge Zaverucha (UFPE) ad hoc

José Garriga Zucal (Universidad de Buenos Aires, Argentina) ad hoc

José Miguel Quedi Martins (UFRGS) ad hoc

José Szwako (Unicamp) ad hoc

Juan Claudio Epsteyn (Ibmec-RJ) ad hoc

Kai Lehmann (PUC-RJ) ad hoc

Kai Michael Kenkel (PUC-RJ) ad hoc

Leonardo Carvalho Braga (PUC-RJ) ad hoc

Ligia Luchmann (UFSC) ad hoc

Lilian Cristina Bernardo Gomes (UFMG) ad hoc

Luciano Da Ros (UFRGS) ad hoc

Ludmila Mendonça Lopes Ribeiro (FGV) ad hoc

Luís Antônio Francisco de Sousa (Unesp) ad hoc

Luis Felipe Miguel (UnB)

Luis Fernando Ayerbe (Unesp) ad hoc

Luiz Antônio Machado (UFRJ, UERJ) ad hoc

Marcelo de Almeida-Medeiros (UFPE) ad hoc

Marcia da Silva Mazon (UFSC) ad hoc 
Marco Aurélio Cepik (UFRGS)

Marcos Del Roio (Unesp) ad hoc

Marcus Faro de Castro (UnB) ad hoc

Marcus Ianoni (UFF) ad hoc

Marcus Melo (UFPE) ad hoc

Maria do Carmo Alves de Albuquerque (Uniban) ad hoc

Maria do Socorro Sousa Braga (Ufscar) ad hoc

Maria Izabel Noll (UFRGS) ad hoc

Maria Thereza Rosa Ribeiro (Ufpel) ad hoc

Marta Arretche (USP)

Marta Mendes da Rocha (PUC-MG) ad hoc

Mónica Salomón (UFSC) ad hoc

Mylene Nogueira Teixeira (UCAM) ad hoc

Nelson Eugênio Pinheiro Montenegro (UFRB) ad hoc

Odílio Alves Aguiar (UFCE) ad hoc

Osmir Dombrowski (Unioeste) ad hoc

Paolo Ricci (USP) ad hoc

Pedro Hermílio Villas Bôas Castelo Branco (UFF) ad hoc

Pedro José Floriano Ribeiro (Ufscar) ad hoc

Pedro Robson Pereira Neiva (Cebrap) ad hoc

Rafael Machado Madeira (PUC-RS) ad hoc

Rafael Wowk (Université Paris 1 - Sorbonne) ad hoc

Rebecca Abers (UnB) ad hoc

Reginaldo Carmello Corrêa de Moraes (Unicamp) ad hoc

Rejane Carvalho (UFC) ad hoc

Riberti de Almeida Felisbino (Unesp) ad hoc

Ricardo Costa de Oliveira (UFPR)

Ricardo Luiz de Souza (Centro Universitário de Sete Lagoas) ad hoc

Rodolfo Liberato de Noronha (UFF) ad hoc

Rodolpho Talaisys Bernabel (USP) ad hoc

Rodrigo Duarte Fernandes dos Passos (UFPI) ad hoc

Rodrigo Ghiringhelli de Azevedo (PUC-RS) ad hoc

Rodrigo Horochovski (UFPR) ad hoc

Rodrigo Patto Sá Motta (UFMG) ad hoc

Ronaldo Bernardino Colvero (Unipampa) ad hoc

Rosangela Marione Schulz (Ufpel) ad hoc

Rosemary Dore (UFMG) ad hoc

Samira Kauchakje (PUC-PR) ad hoc

Sarah Escorel (Fiocruz) ad hoc

Sérgio Soares Braga (UFPR)

Simone Diniz (Ufscar) ad hoc

Teresa Cristina Schneider Marques (Universidade de Cuiabá) ad hoc

Thamy Pogrebinschi (UERJ) ad hoc

Valeria Fernández Hasan (Instituto de Ciencias Humanas, Sociales y Ambientales - Argentina) ad hoc

Valmir Lopes de Lima (UFC) ad hoc

Vanessa Elias de Oliveira (Ufabc) ad hoc

Victor Novicki (UCP) ad hoc

Vilma Soares de Lima Barbosa (UECG) ad hoc

Wagner Pralon Mancuso (USP) ad hoc

Wilson José Ferreira de Oliveira (Ufpel) ad hoc

Yan de Souza Carreirão (UFSC) ad hoc 